\title{
Crowdsourcing Methods to Enhance HIV and Sexual Health Services: A Scoping Review and Qualitative Synthesis
}

\author{
Dan Wu, PhD, ${ }^{a, b}$ Jason J. Ong, MD, PhD, ${ }^{a, c}$ Weiming Tang, PhD, ${ }^{\text {de.f }}$ Tiarney D. Ritchwood, PhD, ${ }^{g}$ \\ Jennifer S. Walker, MLS, ${ }^{h}$ Juliet Iwelunmor, PhD, ${ }^{i}$ and Joseph D. Tucker, MD, PhD ${ }^{a, b, d}$
}

\begin{abstract}
Background: Crowdsourcing is increasingly used to improve community engagement in HIV and sexual health research. In this scoping review, we reviewed studies using crowdsourcing approaches in HIV and sexual health research to identify strengths, opportunities for expansion, and limitations of such approaches.
\end{abstract}

Methods: We searched CINAHL, Web of Science, Embase, and PubMed. Studies were included if they involved crowdsourcing activities, were in the field of HIV or sexual health, and described the methodology in sufficient detail. We conducted a qualitative synthesis of eligible articles.

Results: Our search strategy yielded 431 nonduplicate articles. After screening, 16 articles met the inclusion criteria, including 4 publications that described research from high-income countries, 7 from middle-income countries, 1 from a low-income country, and 4 that had a global focus. There were 4 categories of crowdsourcing: open contests, hackathons, open forums, and incident reporting systems. We identified common phases for data acquisition and dissemination: (1) preparation; (2) problem framing and crowd solicitation; (3) judging submissions; and (4) sharing selected submissions. Strengths of using crowdsourcing approaches include greater innovation due to crowd heterogeneity, encouragement of multisectoral collaboration, empowerment of vulnerable populations,

Received for publication September 8, 2019; accepted September 11, 2019. From the ${ }^{\mathrm{a}}$ Faculty of Infectious and Tropical Diseases, London School of Hygiene and Tropical Medicine, London, United Kingdom; 'bocial Entrepreneurship to Spur Health (SESH) Global, Guangzhou, China; ${ }^{\mathrm{c} C e n t r a l}$ Clinical School, Monash University, Melbourne, Australia; dUniversity North Carolina at Chapel Hill, Project-China, Guangzhou, China; 'Dermatology Hospital, Southern Medical University, Guangzhou, China; ${ }^{\mathrm{f} S c h o o l}$ of Public Health, Southern Medical University, Guangzhou, China; ${ }^{g}$ Family Medicine and Community Health, Duke University,

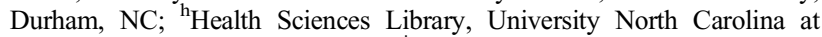
Chapel Hill, Chapel Hill, NC; and ${ }^{\mathrm{i}}$ College for Public Health and Social Justice, Saint Louis University, St. Louis, MO.

This study received support from the National Institutes of Health (NIAID 1R01AI114310, NIAID K24AI143471, NICHD UG3HD096929), the UNCSouth China STD Research Training Center, the UNC Center for AIDS Research (NIAID 5P30AI050410), and the Academy of Medical Sciences and the Newton Fund (NIFVR11181020). UNICEF/UNDP/World Bank/WHO Special Programme for Research and Training in Tropical Diseases (TDR).

The authors have no funding or conflicts of interest to disclose.

D.W. and J.J.O. contributed equally to this work.

Supplemental digital content is available for this article. Direct URL citations appear in the printed text and are provided in the HTML and PDF versions of this article on the journal's Web site (www.jaids.com).

Correspondence to: Joseph D. Tucker, MD, PhD, UNC Project-China, Guangdong Provincial STD Control Department, 2 Lujing Road, Guangzhou 510095, China (e-mail: jdtucker@med.unc.edu).

Copyright ( 2019 Wolters Kluwer Health, Inc. All rights reserved. cost-effectiveness, and relevance to local cultures and styles. Weaknesses among some methods include reliance on the internet, temporal transience, and difficulty in sustaining long-term community engagement.

Conclusions: Crowdsourcing may be useful for HIV implementation research. Further research on crowdsourcing related to HIV and sexual health is needed.

Key Words: crowdsourcing, methods, qualitative, review, HIV

(J Acquir Immune Defic Syndr 2019;82:S271-S278)

\section{INTRODUCTION}

The World Health Organization recognizes community engagement as a critical element in the response to the HIV pandemic. ${ }^{1}$ Community-driven approaches allow stakeholders to identify problems, generate solutions, and share responsibility for implementing interventions. Crowdsourcing, the process of aggregating a group of nonexperts and experts to solve a problem, is increasingly used in public health. ${ }^{2}$ Crowdsourcing is based on the principle of collective wisdom and open access. Collective wisdom suggests that in some contexts, crowds as a whole may out-perform the most experienced individuals within them. ${ }^{3}$ Collective wisdom has been used in political science, philosophy, social science, business, and medicine. ${ }^{3}$

Programs that promote HIV prevention and sexual health may benefit from crowdsourcing because such approaches could bring large groups of people together in new and creative ways to find culturally appropriate solutions to problems. Allowing a diversity of voices to shape health interventions may guard against group-think and limit less effective top-down, homogeneous interventions. ${ }^{4}$ Crowdsourcing approaches may also save time and costs and could speed up finding innovative solutions. ${ }^{5}$ Examples of the ways in which crowdsourcing have been used in HIV prevention include logos for an AIDS conference, ${ }^{6}$ videos to increase HIV testing, ${ }^{7}$ and campaigns for promoting HIV testing services. ${ }^{8}$ Crowdsourcing approaches have been utilized in research for key populations, including in sexual health campaigns for youth $^{6}$ and men who have sex with men (MSM). ${ }^{7-9}$

To date, there are no crowdsourcing reviews focused on HIV and sexual health, despite the increasing use of this approach in these fields. ${ }^{4,5,10,11}$ The purpose of this study is to conduct a qualitative synthesis of existing evidence on the use 
of crowdsourcing approaches in HIV and sexual health promotion and to evaluate the advantages and disadvantages of using crowdsourcing.

\section{METHODS}

We used Arksey and O'Malley's framework for conducting this scoping review. ${ }^{12}$ Scoping studies summarize key evidence on a topic but do not undergo the process of a formal systematic review. We established a list of guiding questions for this review:

1. How did authors define crowdsourcing?

2. What was the purpose and scale of the crowdsourcing approach?

3. What were the types of crowdsourcing approaches?

4. What were the phases or structure of crowdsourcing approaches?

5. What were the strengths and weaknesses of crowdsourcing approaches?

We searched CINAHL, Web of Science, Embase, and PubMed on July 16, 2018, identifying articles published that reported crowdsourcing activities related to HIV and sexual health. The literature search included Medical Subject Headings (MeSH), Emtree headings, and related text and keyword searches. Search terms included sexual health-related terms such as HIV, sexually transmitted infection(s), sexual behavior, or condom use. Eligibility criteria included the following: study design/contents involving crowdsourcing activities; study topics in the field of HIV or sexual health; and methodologies described in sufficient detail. A full search strategy is included in Appendix A, Supplemental Digital Content, http://links.lww.com/QAI/B383. We used PRISMAScR checklist (see Appendix B, Supplemental Digital Content, http://links.lww.com/QAI/B384) to report this review. Two members of the research team, J.J.O. and D.W., independently reviewed manuscript titles and abstracts according to the eligibility criteria. After screening the titles and abstracts, eligibility of relevant citations were further reviewed independently by the 2 researchers. Discrepancies and uncertainties were discussed with the research group until agreement was achieved. Eligible full articles were included for qualitative synthesis.

\section{RESULTS}

Our search strategy yielded 431 nonduplicate articles, and 48 manuscripts were fully reviewed. Among the 48 citations, 32 were excluded: 10 had insufficient detail on methods, 16 either did not use crowdsourcing or were not about sexual health, 3 were duplicates, 2 were study protocols, and 1 was a post-hoc analysis of existing ideas from social media platforms. We identified 16 full articles for qualitative synthesis. Table 1 summarizes the aims, methods, and participants in the included studies. Four publications reported research from high-income countries, ${ }^{9,13-15} 7$ from middle-income countries, $7,8,16-211$ from a low-income country, ${ }^{22}$ and 4 had a global focus. ${ }^{4,6,18,23}$ They focused on HIV, ${ }^{6-9,13,17,19,22,23}$ sexual health promotion, ${ }^{14-16,18,20,21}$ and sexual violence prevention. ${ }^{14-16}$ A total of 11 used or evaluated crowdsourcing contests. The geographic scale of crowdsourcing activities included citywide, $, 13,17,19,20$ nationwide, ${ }^{7,8,14-16,18,21}$ regional, $^{22}$ and global. ${ }^{4,6,18,23}$

\section{Definition of Crowdsourcing}

Thirteen studies clearly defined their approaches as crowdsourcing. ${ }^{4,6-9,13,16-21,23}$ Three studies did not have an explicit definition of crowdsourcing in their study, yet their approach was consistent with the principles of crowdsourcing. ${ }^{14,15,22}$ Based on our review, we defined crowdsourcing as the process of inviting a group of individuals to complete a task, attempt to solve a problem, or develop innovative ideas. In 10 cases, individuals from diverse backgrounds were invited without restriction (ie, anyone with access to the invite was allowed to take part). ${ }^{4,6-9,16,18-21}$ In 6 studies, the crowd was more narrowly defined around a target population. ${ }^{13-15,17,22,23}$ They shared their original ideas to answer questions or propose solutions.

\section{Categories of Crowdsourcing}

We identified 4 categories of crowdsourcing activities, including open contests, hackathons, open forums, and incident reporting systems. Open contests, also called innovation challenges or innovation contests, are a subset of crowdsourcing approaches that solicit creative contributions from the target populations, evaluate submissions, celebrate finalists, and share the finalist ideas. Open contests were the most common crowdsourcing model used for HIV and sexual health research. Open contests have been used to invite target communities' contribution to design ideas, images, and videos on how to promote condom use and HIV testing. ${ }^{6,8,9,18-21,24}$ Hackathons are intensive, approximately 72-hour contests that bring people together to complete a specific task. For example, a hackathon could help develop HIV test promotion campaigns. ${ }^{8,17}$ For both open contests and hackathons, entries were evaluated according to preselected criteria, and then, several finalists were selected to receive prizes. An open forum refers to Wiki-like platforms for individuals to contribute to a topic of interest. For example, the open-source medical record system, OpenMRS, invites implementers and developers to communicate problems and solutions through the system. ${ }^{22}$ An incident reporting system refers to an established online system that allows survivors or witnesses to report relevant incidents to a shared platform that is accessible by the public. Our review found past incident report and immediate report systems for sexual abuse or sexual harassment experiences. The former allowed adult victims who were abused in their childhood to voluntarily and describe what had happened to them and express their opinions or suggestions. ${ }^{14,15}$ The latter was an immediate incident reporting system that allowed sexual harassment victims or witnesses to submit an immediate report to a platform through SMS text messages or social media tools about their experiences, which then plots the locations where the sexual harassment incident occurred. ${ }^{16}$ 
TABLE 1. Summary of the Aims, Methods, and Participants in Studies Included in the Review

\begin{tabular}{|c|c|c|c|c|}
\hline Author & $\begin{array}{c}\text { Publication } \\
\text { Year }\end{array}$ & $\begin{array}{c}\text { Geographic } \\
\text { Location }\end{array}$ & Aim & $\begin{array}{l}\text { Category } \\
\text { Crowdsourc }\end{array}$ \\
\hline $\begin{array}{l}\text { Hildebrand } \\
\text { et } \mathrm{al}^{23}\end{array}$ & 2013 & Global & $\begin{array}{l}\text { To develop a strategy for how to better } \\
\text { engage young people in decision- } \\
\text { making processes on AIDS }\end{array}$ & Open contest \\
\hline $\begin{array}{c}\text { Mathews } \\
\text { et } \mathrm{al}^{9}\end{array}$ & 2017 & US & $\begin{array}{l}\text { To evaluate the feasibility of using } \\
\text { a crowdsourcing contest to promote } \\
\text { HIV cure research community } \\
\text { engagement }\end{array}$ & Open contest \\
\hline $\begin{array}{r}\text { Mathews } \\
\text { et }^{1}{ }^{13}\end{array}$ & 2018 & US & $\begin{array}{l}\text { To examine the feasibility of using } \\
\text { crowdsourcing contests as a method of } \\
\text { HIV cure research engagement }\end{array}$ & Open contest \\
\hline
\end{tabular}

\begin{tabular}{|c|c|c|c|c|}
\hline Ong et al ${ }^{15}$ & 2017 & Global & $\begin{array}{l}\text { To evaluate a crowdsourced logo contest } \\
\text { as part of an international HIV } \\
\text { conference }\end{array}$ & Open contest \\
\hline Pan et $\mathrm{al}^{4}$ & 2017 & Global & $\begin{array}{l}\text { To evaluate the effectiveness and cost of } \\
\text { health-focused innovation design } \\
\text { contests }\end{array}$ & Open contest \\
\hline Peuchaud ${ }^{16}$ & 2014 & Egypt & $\begin{array}{l}\text { To describe a case study of how social } \\
\text { media activists have harnessed the } \\
\text { power of Facebook, Twitter, and } \\
\text { mobile phone networks to address } \\
\text { sexual harassment }\end{array}$ & $\begin{array}{l}\text { Incident reporting } \\
\text { system }\end{array}$ \\
\hline $\begin{array}{l}\text { Rassenhofer } \\
\text { et } \mathrm{al}^{15}\end{array}$ & 2015 & Germany & $\begin{array}{l}\text { To discuss the feasibility and value of } \\
\text { using a critical incident reporting } \\
\text { system sponsored by the institution } \\
\text { responsible versus one sponsored by an } \\
\text { independent governmental body }\end{array}$ & $\begin{array}{l}\text { Incident reporting } \\
\text { system }\end{array}$ \\
\hline $\begin{array}{l}\text { Rassenhofer } \\
\text { et } \mathrm{al}^{14}\end{array}$ & 2013 & Germany & $\begin{array}{l}\text { To analyze the experiences of now-adult } \\
\text { victims of past sexual abuse and make } \\
\text { recommendations for policy changes } \\
\text { and legislations }\end{array}$ & $\begin{array}{l}\text { Incident reporting } \\
\text { system }\end{array}$ \\
\hline $\begin{array}{l}\text { Seebregts } \\
\text { et } \mathrm{al}^{22}\end{array}$ & 2009 & Africa & $\begin{array}{l}\text { To develop an open-source electronic } \\
\text { medical record application, to provide } \\
\text { regional support for application } \\
\text { implementations }\end{array}$ & Open forum \\
\hline Tang et $\mathrm{al}^{7}$ & 2016 & China & $\begin{array}{l}\text { To compare first-time HIV testing rates } \\
\text { among MSM and transgender } \\
\text { individuals who received } \\
\text { a crowdsourced or a health marketing } \\
\text { HIV test promotion video }\end{array}$ & Open contest \\
\hline Tang et $\mathrm{al}^{8}$ & 2018 & China & $\begin{array}{l}\text { To evaluate the effect of a crowdsourced } \\
\text { HIV intervention on HIV testing } \\
\text { uptake among MSM }\end{array}$ & $\begin{array}{l}\text { Open contest and } \\
\text { hackathon }\end{array}$ \\
\hline Tucker et al ${ }^{18}$ & 2018 & China & $\begin{array}{l}\text { To describe a crowdsourcing designathon, } \\
\text { summarize outputs, and discuss } \\
\text { implications for public health }\end{array}$ & Hackathon \\
\hline Tucker et al ${ }^{18}$ & 2018 & Global & $\begin{array}{l}\text { To describe crowdsourcing contests by } \\
\text { stage, examines ethical problems at } \\
\text { each stage, and proposes potential } \\
\text { ways of mitigating risk }\end{array}$ & Open contest \\
\hline
\end{tabular}
as part of an international HIV health-focused innovation design media activists have harnessed the discuss the feasibility and value of using a critical incident reporting responsible versus one sponsored by an analyze the experiences of now-adult victims of past sexual abuse and make policy changes

develop an open-source electroni regional support for application among MSM and transgender individuals who received a crowdsourced or a health marketing HIV intervention on HIV testing

\section{Crowd Contribution}

Young people aged 15-29 years from 79 countries. Shared opinions about young people and HIV, including young people living with HIV, access to services, and the legal and social environment, among others.

Submit entries about "What does an HIV cure mean to you?" in the form of videos, images, and audio.

Community members participated in focus-group discussions, shared opinions about their acceptance of a crowdsourcing contest, and developed a contest name, logo, and hashtag.

Participants designed and submitted a conference logo.

Contributed and submitted entries

Individuals who experienced or witnessed sexual harassment reported the incident to the system

Child sexual abuse survivors, confidants or acquaintances of victims, offenders, and people who were concerned about the issue reported the incident or shared thoughts about the topic to the system

Child sexual abuse survivors, confidants or acquaintances of victims, offenders, and people who were concerned about the issue reported the incident or shared thoughts about the topic to the system

The medical record application implementers and developers communicated problems and solutions on the platform

The crowd contributed and submitted HIV test promotion videos

The crowd contributed and submitted HIV test promotion images, texts, and developed an intervention package for a health campaign.

CDC staff, a local gay community member, and other participants developed an intervention package

The crowd contributed and submitted entries on relevant topics 


\begin{tabular}{|c|c|c|c|c|c|}
\hline Author & $\begin{array}{c}\text { Publication } \\
\text { Year }\end{array}$ & $\begin{array}{c}\text { Geographic } \\
\text { Location }\end{array}$ & Aim & $\begin{array}{c}\text { Category of } \\
\text { Crowdsourcing }\end{array}$ & Crowd Contribution \\
\hline Zhang et $\mathrm{al}^{19}$ & 2018 & China & $\begin{array}{l}\text { To examine text entries submitted to } \\
\text { a crowdsourcing contest on the } \\
\text { meaning of an HIV cure }\end{array}$ & Open contest & $\begin{array}{l}\text { Men who have sex with men, people who } \\
\text { injected drugs, people living with HIV, } \\
\text { and local residents contributed and } \\
\text { submitted text entries. }\end{array}$ \\
\hline Zhang et $\mathrm{al}^{21}$ & 2015 & China & $\begin{array}{l}\text { To describe creative contributory contests } \\
\text { to promote sexual health and provide } \\
\text { guidance for designing creative } \\
\text { contributory contests }\end{array}$ & Open contest & $\begin{array}{l}\text { Local populations, including MSM, } \\
\text { university students, graphic designers } \\
\text { and artists contributed and submitted } \\
\text { entries on relevant topics }\end{array}$ \\
\hline Zhang et $\mathrm{al}^{20}$ & 2017 & China & $\begin{array}{l}\text { To increase sexual health awareness } \\
\text { among youth using an innovation } \\
\text { contest and to evaluate community } \\
\text { engagement in the contest }\end{array}$ & Open contest & $\begin{array}{l}\text { Individuals contributed and submitted } \\
\text { images promoting sexual health among } \\
\text { young people. }\end{array}$ \\
\hline
\end{tabular}

MSM, men who have sex with men.

\section{Crowdsourcing Phases}

We identified 4 common phases for crowdsourcing approaches: (1) preparation, (2) problem framing and crowd solicitation, (3) judging submissions, and (4) sharing selected submission(s).

\section{Phase 1: Preparation}

The first phase of many crowdsourcing approaches was preparation. 6,9,14,15,17,18,20,22 Activities included forming steering committees and/or advisory boards that establishes the rules and identifies the problem(s) to be solved, $6,8,14-18,21,23$ with some groups also conducting qualitative research to support the design of the crowdsourcing activity. ${ }^{13}$ All steering committees involved experts and people who have lived experience of the disease. The crowdsourcing activities were led by governmental authorities or other decision-makers, ${ }^{14,15}$ research institutes, ${ }^{6-9,13,16-20,22}$ or nongovernmental organizations. ${ }^{23}$ They initiated, organized, implemented, and monitored the whole activity. Some research groups noted the importance of including individuals from key populations in the steering committee, especially those who are marginalized and vulnerable, because the absence of their voice may reduce the effectiveness and trust in the activity. ${ }^{18,23}$ Regular steering committee meetings were a common approach to follow-up on progress and make refinements. ${ }^{18}$ Workshops, discussions, or other training activities were important strategies to strengthen the relevant parties' capacity and leadership. ${ }^{22}$ One study assessed the feasibility before launching a crowdsourcing contest using focus group interviews with key populations. ${ }^{13}$ Through the feasibility evaluation process, they developed logos, contest names, strategies to engage the community, and other contest promotion materials. ${ }^{13}$

\section{Phase 2: Problem Framing and Crowd Solicitation}

The second phase includes framing the problem and promotion of the activity to the crowd. Online promotion strategies included Facebook, Twitter, Instagram, emails, open Skype calls, Weibo (a popular microblog used in China), WeChat (a Chinese instant messaging mobile application), and official websites. ${ }^{6-9,16,17,19,21,23}$ In-person pro- motional events, such as class lectures, community-based open forums, recreational activities, special events such as the World AIDS Day, were adopted by other studies. ${ }^{9,17,23}$ Local radio, which reaches a large number of people in LMICs, was also used to advertise the activity. ${ }^{9}$ The crowdsourcing approach led by UNAIDS used individual network distribution, encouraging young people connected to sexual health activism to spread the activity information to family, friends, and acquaintances. ${ }^{23}$ In-person engagement events were considered key for soliciting submissions from those who had limited access to the internet. ${ }^{9,21,23}$ One study received more contest submissions from people who attended inperson events compared with those who did not attend inperson events. ${ }^{9}$

Some studies had strictly defined formats for submission. Six studies solicited images, videos, audio files, and texts with predetermined size limits, ${ }^{7-9,13,17,21}$ and 1 study allowed victims or witnesses to immediately report the time, location, and case description to a sexual harassment incident reporting system. ${ }^{16}$ Two studies had relatively flexible formats for submission without limiting the size of the submission to allow submitters to freely express what matter to them. ${ }^{15,23}$

We identified various forms of incentives for participation. For open contests and hackathons, material prizes (eg, iPad, cash, or other gifts) and coverage of participation expenses were awarded. ${ }^{9,17,19,21}$ Nonfinancial benefits such as feeling empowered due to participation, having their voice heard, and contributing to the fight against HIV were also considered important motivators for participation in the crowdsourcing activity. $6,15,16,23$

\section{Phase 3: Evaluating Submissions}

Submissions were judged by crowd and expert judges based on predetermined criteria. ${ }^{6-9,17-21}$ The judging process was mainly to ensure quality of submissions and to select top ones for future interventions, health education, or health advocacy purposes. ${ }^{7,8,17,21}$ All crowdsourcing open contests and hackathons had the judging phase and submissions scored according to the predetermined evaluation criteria. Fairly selecting judges from local key groups was important to increase community ownership of the event and to 
incorporate local preferences. ${ }^{18}$ Using predetermined evaluation criteria helped improve judging consistency across different judges. ${ }^{18}$ Other crowdsourcing models had other forms of evaluation of their crowdsourced data such as analyzing the content of submissions. ${ }^{14-16,20,23}$

\section{Phase 4: Sharing the solution(s)}

All crowdsourcing activities shared their outputs in some way, and the sharing was sometimes nested within the process of inviting the crowd to judge submissions. We defined the outputs of a crowdsourcing activity as outputs of any format, including generation of new knowledge (eg, perceptions of HIV cure), ${ }^{19}$ creation of new products (eg, images, videos, logos, and reporting platforms $)^{6,14-16,21,22}$ or intervention materials (eg, public health intervention materials), ${ }^{7,8,21}$ development of community engagement campaigns (ie, engaging youth in AIDS response), ${ }^{23}$ social media activism (eg, undermining the climate of social acceptability for sexual harassers), ${ }^{16}$ policy changes (eg, institutional priority setting and guidelines for handling sexual abuse and preventing it), ${ }^{15,23}$ adoption of new legislations, ${ }^{14}$ further research and education programs,${ }^{14}$ and change in medical practice. ${ }^{14}$

Some open contests and hackathons shared submissions during the evaluation process, and after, finalists were selected. ${ }^{9}, 17,18,20,21$ Presenting results to governmental authorities or other decision-makers facilitated policy change, and releasing results on websites and press conferences to inform sexual abuse victims about the ongoing process built up people's trust and readiness to use the incident report system. ${ }^{14}$ Incident report data were used to convince shopkeepers, police officers, and restaurant owners to begin intervening once they see sexual harassment in their neighborhoods. When they agreed, a "harassment-free zone" sticker was given to them, illustrating to customers that their business was a harassment-free zone. ${ }^{16}$

\section{Crowdsourcing Activity Evaluation}

Most crowdsourcing activities were evaluated in some way. The first form was to evaluate the crowdsourcing activity itself. Assessments included quantitative data about traffic (the number of webpage visits, followers, and reach of unique users) ${ }^{9}$ and qualitative evaluation of the content of submissions (eg, emerging themes from the text submissions). $6,9,14,15,19,23$ The second form of evaluation focused on examining the level of community engagement as a result of the crowdsourcing activity. One study evaluated community engagement and the impact of participation in an open contest to promote sexual health using qualitative interviews. ${ }^{20}$ They found different forms of engagement activities including submitting an entry to the contest and helping organize the contest events. ${ }^{20}$ Participating in a contest helped increase knowledge in the topic, multisectoral collaboration, and reorientation of professionals toward people-centered sexual health programs. ${ }^{20}$ The third form of evaluation was through randomized control trials to evaluate the effectiveness of crowdsourced intervention materials, compared with conventional sexual health campaigns. For example, 2 studies from China evaluated the effects of crowdsourced HIV testing promotion approaches in men who have sex with men and concluded that it can significantly improve HIV test uptake. ${ }^{7,8}$

\section{Strengths and Weaknesses}

Crowdsourcing in health has strengths and weaknesses (Table 2). There may be higher potential for innovation due to soliciting crowd wisdom, encouraging collaboration from multiple sectors, empowering key/marginalized populations by integrating their perspectives into high-level policymaking process, and creating intervention strategies that are cost-effective and locally relevant with strong potential for scalability. Meanwhile, disadvantages include some crowdsourcing projects only relying on internet channels, which limit participation. Some open contests, although, included a series of in-person events to mitigate such an effect. 9,19-21,23 Formulating a call for entries, which is engaging and widely disseminating, can be challenging. For open contests and hackathons, these approaches are relatively short-term and have potential risks of exclusion of community members from steering committees, biased crowd judging, exploitation and insufficient recognition of contributors, and limited sharing. ${ }^{18}$

TABLE 2. The Strengths and Weaknesses of Crowdsourcing Approaches

\begin{tabular}{|c|c|}
\hline & Articles \\
\hline \multicolumn{2}{|l|}{ Strengths } \\
\hline $\begin{array}{l}\text { Higher potential for innovation due to heterogeneity } \\
\text { of knowledge in the crowd compared with a panel } \\
\text { of experts }\end{array}$ & 21 \\
\hline $\begin{array}{l}\text { Encourages collaboration between different groups, } \\
\text { fields, and sectors }\end{array}$ & $9,21,22,32,33$ \\
\hline Empowers key populations & $6,7,17,21,23,33$ \\
\hline Builds community capacity & 16,22 \\
\hline $\begin{array}{l}\text { Integrates grassroots perspectives into high-level } \\
\text { strategy and policy process }\end{array}$ & $14,15,23$ \\
\hline $\begin{array}{l}\text { Minimal cost compared with social marketing for } \\
\text { public health intervention development }\end{array}$ & $6,7,17,22$ \\
\hline $\begin{array}{l}\text { Creates messages/strategies that are locally relevant } \\
\text { and feasible to implement }\end{array}$ & $6,7,17,22$ \\
\hline $\begin{array}{l}\text { Documents events of interests; mitigates the fear of } \\
\text { stigma and retaliation through anonymous reporting }\end{array}$ & $14-16$ \\
\hline $\begin{array}{l}\text { Strong scalability and wide coverage of key } \\
\text { populations }\end{array}$ & 16,22 \\
\hline \multicolumn{2}{|l|}{ Weaknesses } \\
\hline $\begin{array}{l}\text { Over-reliance on internet channels and ignores } \\
\text { individuals who lack internet access }\end{array}$ & $9,18,21$ \\
\hline $\begin{array}{l}\text { Risk of too few submissions if the topics are } \\
\text { nonsensitive to populations of interest }\end{array}$ & 21 \\
\hline $\begin{array}{l}\text { Open contests and hackathons are temporally transient } \\
\text { and relatively short term }\end{array}$ & $9,18,21$ \\
\hline $\begin{array}{l}\text { Open contests have potential risks of excluding } \\
\text { community members from steering committee, } \\
\text { biased crowd judging }\end{array}$ & $9,18,21$ \\
\hline $\begin{array}{l}\text { Open contests and hackathons are subject to } \\
\text { exploitation and insufficient recognition of } \\
\text { contributors and limited sharing }\end{array}$ & $9,18,21$ \\
\hline $\begin{array}{l}\text { Incident report systems focuses on collecting } \\
\text { survivors' experiences of sexual abuse/harassment } \\
\text { but not directly preventing it from happening }\end{array}$ & $14-16$ \\
\hline
\end{tabular}


TABLE 3. Crowdsourcing for HIV and Sexual Health Implementation Science Based on the RE-AIM Framework ${ }^{25}$

\begin{tabular}{|c|c|}
\hline & Potential Role for Crowdsourcing \\
\hline Reach & $\begin{array}{l}\text { 1. By using local and audience-friendly language suited } \\
\text { for specific contexts, crowdsourcing can reach } \\
\text { individuals from communities who may have } \\
\text { language barriers. }\end{array}$ \\
\hline & $\begin{array}{l}\text { 2. Crowdsourcing engages at-risk targets groups during } \\
\text { intervention development and evaluation, increasing } \\
\text { the potential reach of the intervention. }\end{array}$ \\
\hline
\end{tabular}

Efficacy HIV and sexual health interventions developed using crowdsourcing methods have increased HIV test uptake rates among MSM.

Adoption

Implementation

Maintenance
Crowdsourcing may increase adoption of an intervention by including key stakeholders, including key populations, in decision-making roles.

1. At the individual level, crowdsourcing may be easier to implement because it draws on local user preferences, styles, and culture that are known to influence implementation.

2. From an implementer perspective, an intervention designed for the local health system may be easier to implement.

1. From the individual perspective, crowdsourcing may increase trust in health services ie, known to be important for maintaining an intervention over time.

2. Crowdsourcing activities also have strong community ownership, resulting in more active engagement in maintaining or scaling a program.
HIV Examples and References

CrowdOutAIDS led by UNAIDS organized open forums in all languages spoken in the BRICS countries (Brazil, Russia, India, China, and South Africa) to minimize language difficulties. ${ }^{23}$

1. CrowdOutAIDS reached 3295 youth participants from different regions worldwide online and 1605 participants offline. ${ }^{23}$

2. Over 14 weeks, the in-person community events of an HIV cure open contest reached 144 participants in North Carolina. The online promotion channels reached 80,624 unique users. ${ }^{9}$

3. A crowdsourcing intervention study to promote the HIV test among Chinese MSM showed that their messages reached $91.4 \%$ of men recruited in the study, and $67.1 \%$ of them shared at least one image or text. ${ }^{34}$

1. One RCT showed that, compared with the control period, the crowdsourcing intervention significantly improved HIV test uptake rate among Chinese gay men, especially HIV self-testing. ${ }^{8}$

2. Another RCT comparing the effects of crowdsourced HIV test promotion videos and expert driven health marketing videos found that the former had similar effects on improving HIV testing but higher community engagement?

1. The child sexual abuse incident reporting program involved both church organizations and government in the program development process. This activity resulted in more actions against child sexual abuse by both churches (ie, adopting guidelines and framework for preventing and managing child sexual abuse, as well as research project on the topic) and the government (ie, amending laws to strengthen victims' rights). ${ }^{14,15}$

1. A RCT evaluating the effectiveness of a crowdsourcing intervention on HIV test uptake among an MSM sample showed that $62 \%$ of them tested for HIV during the study period. ${ }^{8}$

2. Following the guidance from the study team, local CDC and gay community organizations in all 8 study sites in an intervention program implemented a story contest with contextual adaptations to promote HIV testing among local MSM, ${ }^{8}$ which resulted in increased likelihood of facility-based HIV testing behaviors. ${ }^{34}$

1. In a crowdsourcing intervention study, among MSM participants, the proportion who maintained HIV testing every 3 months after the intervention ranged from $32.0 \%$ to $39.4 \%$ at the end of the 12-month study, compared with $19.1 \%-29.0 \%$ before the intervention. ${ }^{8}$

2. The open-source electronic medical record application (OpenMRS) was established and applied to HIV/AIDS and tuberculosis patients and treatment information management in developing countries. This system enabled local implementers and developers to freely collaborate and discuss technical issues pertaining to the development of OpenMRS system. This model has been maintained since its first establishment in South Africa in 2006 and replicated in several other African sites. ${ }^{22}$

R: Reach, ie, proportion of the target population that participated. E: Efficacy, ie, success rate at changing desired outcomes. A: Adoption, ie, proportion of target settings involved. I: Implementation, ie, extent to which the program was delivered as intended. Measures of implementation have both individual and program levels. Measures of individual-level implementation include participant follow-through or "adherence" to regimens, whereas measures of program-level implementation mainly include to what extent staff members deliver the intervention as intended. M: Maintenance, ie, extent to which the program outcomes (at the individual level) and program availability (at the setting level) were sustained over time. MSM, men who have sex with men. 


\section{Crowdsourcing for Implementation Science}

Based on the RE-AIM framework, ${ }^{25}$ we analyzed how crowdsourcing may contribute to implementation science. RE-AIM is a model commonly used to evaluate the impact of public health interventions in 5 dimensions: reach, efficacy, adoption, implementation, and maintenance. ${ }^{25}$ Crowdsourcing may be useful for several parts of implementation research (Table 3). Crowdsourcing may increase the reach of interventions by engaging large groups of local at-risk individuals in the development and evaluation of the intervention itself. ${ }^{23}$ Crowdsourcing may increase adoption of an intervention by including key policymakers, implementers, and key populations in decision-making roles. ${ }^{14,15}$ Crowdsourcing programs could be easier to implement because it draws on local styles, preferences, and culture, which are known to influence implementation. ${ }^{8}$ Regarding maintenance, crowdsourcing often enhances trust in health services, which is important for maintaining an intervention over time, and can create strong community ownership, resulting in more active engagement in sustaining or scaling up programs. ${ }^{22}$

\section{DISCUSSION}

We conducted a scoping review and qualitatively examined studies utilizing crowdsourcing in HIV and sexual health research to identify ways in which previous studies applied crowdsourcing methods and identify strengths and limitations of such approaches. Given that crowdsourcing methods are used increasingly in HIV and sexual health research, it may be worthwhile to create a common framework for ensuring quality in designing and reporting future crowdsourcing studies. A practical guide for conducting open contests was published by the World Health Organization Special Programme on Training and Research in Tropical Diseases. $^{24}$ The emergence of common phases in crowdsourcing activities in our review further supports the potential for creating such a framework.

Researchers used a variety of crowdsourcing approaches to address HIV and sexual health challenges across different geographical and cultural contexts. Open contests, in conjunction with hackathons, were the most commonly used crowdsourcing model to develop cost-effective public health intervention programs. This approach is particularly appealing in HIV research because it enables large groups of people who are often marginalized or difficult to reach to create culturally appropriate solutions to problems facing their communities. Crowdsourcing is thus a practical means for effective community engagement, ${ }^{26}$ which is recognized by the World Health Organization as a key component to the HIV response. ${ }^{1}$ Although less commonly used, an incident reporting system was another way to harness the wisdom of the crowds in a timely manner, for instance, the real-time reporting platform to document sexual harassment incidents. ${ }^{16} \mathrm{~A}$ similar approach has also been implemented to protect sex workers from dangerous clients, demonstrating the capacity for scaling this up in other nations. ${ }^{27}$

Our review has demonstrated several strengths of using crowdsourcing methods in HIV and sexual health. Multidisci- plinary collaboration and heterogeneity are key features, which maximize the potential for innovations by aggregating crowd wisdom. Another advantage is that crowdsourcing activities provide an anonymous channel for people to make their voice heard without fearing stigma. This illustrates the potential of crowdsourced approaches in empowering marginalized or vulnerable communities and integrating their perspectives into priority setting as well as facilitating people-centered policymaking to tackle stigmatized sexual health problems.

Our scoping review also identified potential weaknesses of crowdsourcing that can be improved. One major concern associated with crowdsourcing activities is that they often rely on having internet access and thus constrain participation ${ }^{23}$; however, in-person engagement events have been used to mitigate this risk. ${ }^{13,23,28}$ A combination of online and inperson promotional strategies may help to reach those who might be difficult to approach in-person as well as to reach those who have difficulty accessing the internet. ${ }^{18,21}$ In addition, open contests are relatively short-term, and it is challenging to sustain community engagement. Nevertheless, a series of sequential short-term contests may help sustain interest and engagement. ${ }^{29}$ Open forums for developers and implementers of an open-source medical record system to communicate and the immediate incident reporting system that allows sexual harassment victims or witnesses to submit an immediate report are good crowdsourcing examples that can sustain engagement. The former started in 2006, whereas the latter started in 2010. Both are ongoing programs and have been expanded to a wider international community. ${ }^{30,31}$

Our review has implications for implementation science and the standardization of crowdsourcing methods. Our data suggest that crowdsourcing may be useful for several components of implementation science. Our synthesized data suggest that there are some cross-cutting themes in crowdsourcing research studies. The common phases that we identified in this review may serve as the basis for subsequent standardization of crowdsourcing methods. The WHO/TDR Practical Guide on Crowdsourcing in Health and Health Research provides another foundation, but more formal guidance is needed.

\section{CONCLUSIONS}

Crowdsourcing methods are increasingly used in HIV and sexual health research. They include a diverse set of activities but share common phases with one another. These common elements may be useful for producing a common framework for designing and reporting future crowdsourcing projects. Further research related to crowdsourcing in the context of implementation science is needed.

\section{REFERENCES}

1. World Health Organization community engagement framework for quality, people-centred and resilient health services. Available at: http://apps.who.int/iris/bitstream/handle/10665/259280/WHO-HIS-SDS2017.15-eng.pdf;jsessionid=E4B7F496187C452F4C27BFB897E359E9? sequence=1. Accessed August 20, 2018.

2. Tucker JD, Day S, Tang WM, et al. Crowdsourcing in medical research: concepts and applications. PeerJ. 2019;7:e6762.

3. Surowiecki J. The Wisdom of Crowds. New York, NY: Anchor Books; 2005 . 
4. Pan SW, Stein G, Bayus B, et al. Systematic review of innovation design contests for health: spurring innovation and mass engagement. $B M J$ Innov. 2017;3:227-237.

5. Crequit $\mathrm{P}$, Mansouri G, Benchoufi M, et al. Mapping of crowdsourcing in health: systematic review. J Med Internet Res. 2018;20:e187.

6. Ong JJ, Bilardi JE, Tucker JD. Wisdom of the crowds: crowd-based development of a logo for a conference using a crowdsourcing contest. Sex Transm Dis. 2017;44:630-636.

7. Tang W, Han L, Best J, et al. Crowdsourcing HIV test promotion videos: a noninferiority randomized controlled trial in China. Clin Infect Dis. 2016;62:1436-1442.

8. Tang W, Wei $\mathrm{C}, \mathrm{Cao} \mathrm{B}$, et al. Crowdsourcing to expand HIV testing among men who have sex with men in China: a closed cohort stepped wedge cluster randomized controlled trial. PLoS Med. 2018;15:e1002645.

9. Mathews A, Farley S, Blumberg M, et al. HIV cure research community engagement in North Carolina: a mixed-methods evaluation of a crowdsourcing contest. J Virus Erad. 2017;3:223-228.

10. Swan M. Crowdsourced health research studies: an important emerging complement to clinical trials in the public health research ecosystem. $J$ Med Internet Res. 2012;14:e46.

11. Hossain M, Kauranen I. Crowdsourcing: a comprehensive literature review. Strateg Outsourc Int J. 2015;8:2-22.

12. Arksey H, O'Malley L. Scoping studies: towards a methodological framework. Int J Soc Res Methodol. 2005;8:19-32.

13. Mathews A, Farley S, Hightow-Weidman L, et al. Crowdsourcing and community engagement: a qualitative analysis of the 2BeatHIV contest. $J$ Virus Erad. 2018;4:30-36.

14. Rassenhofer M, Spröber N, Schneider T, et al. Listening to victims: use of a critical incident reporting system to enable adult victims of childhood sexual abuse to participate in a political reappraisal process in Germany. Child Abuse Neglect. 2013;37:653-663.

15. Rassenhofer M, Zimmer A, Spröber N, et al. Child sexual abuse in the Roman Catholic Church in Germany: comparison of victim-impact data collected through church-sponsored and government-sponsored programs. Child Abuse Neglect. 2015;40:60-67.

16. Peuchaud S. Social media activism and Egyptians' use of social media to combat sexual violence: an HiAP case study. Health Promot Int. 2014; 29(suppl 1):i113-120.

17. Tucker J, Tang W, Li H, et al. Crowdsourcing designathon: a new mode for multisectoral collaboration. Ann Glob Health. 2017;83:166-167.

18. Tucker JD, Pan SW, Mathews A, et al. Ethical concerns of and risk mitigation strategies for crowdsourcing contests and innovation challenges: scoping review. J Med Internet Res. 2018;20:e75.

19. Zhang A, Pan X, Wu F, et al. What would an HIV cure mean to you? Qualitative analysis from a crowdsourcing contest in Guangzhou, China. AIDS Res Hum retroviruses. 2018;34:80-87.
20. Zhang W, Schaffer D, Tso LS, et al. Innovation contests to promote sexual health in China: a qualitative evaluation. BMC Public Health. 2017;17:78

21. Zhang Y, Kim JA, Liu F, et al. Creative contributory contests to Spur innovation in sexual health: 2 cases and a guide for implementation. Sex Transm Dis. 2015;42:625-628.

22. Seebregts CJ, Mamlin BW, Biondich PG, et al. The OpenMRS implementers network. Int J Med Inform. 2009;78:711-720.

23. Hildebrand M, Ahumada C, Watson S. CrowdOutAIDS: crowdsourcing youth perspectives for action. Reprod Health Matters. 2013;21:57-68.

24. TDR. Crowdsourcing in health and healthresearch: a practical guide. Geneva, Switzerland: World Health Organization; 2018. TDR/STRA/ 18.4. Available at: https://www.who.int/tdr/publications/year/2018/ crowdsourcing-practical-guide/en/.

25. Glasgow RE, Vogt TM, Boles SM. Evaluating the public health impact of health promotion interventions: the RE-AIM framework. Am J Public Health. 1999;89:1322-1327.

26. US Department of Health and Human Services. Principles of Community Engagement. Available at: https://www.atsdr.cdc.gov/communityengagement/ pdf/PCE_Report_508_FINAL.pdf. Accessed Aug 20, 2018.

27. Ugly Mugs: The Technology Saving the Lives of Sex Workers. Available at: https://www.theguardian.com/voluntary-sector-network/2017/may/ 10/national-ugly-mugs-the-technology-saving-the-lives-of-sex-workers. Accessed March 16, 2019.

28. Zhang Y, Tang S, Li K, et al. Quantitative evaluation of an innovation contest to enhance a sexual health campaign in China. BMC Infect Dis. 2019;19:112.

29. Mathews A. "Crowdsourcing Is When the Public Collaborates to Solve Problems." 2017. Available at: https://2beathiv.org/2beathiv-contests/. Accessed November 9, 2018.

30. OpenMRS. The Global OpenMRS Community Works Together to Build the World's Leading Open Source Enterprise Electronic Medical Record System Platform. 2019. Available at: https://openmrs.org/. Accessed March 20, 2019.

31. HarassMap. HarassMap: Stop Sexual Harassment Together. 2019. Available at: https://harassmap.org/en/who-we-are/. Accessed March 20, 2019.

32. Group SS, Tucker JD. Crowdsourcing to promote HIV testing among MSM in China: study protocol for a stepped wedge randomized controlled trial. Trials. 2017;18:447.

33. Zhang T, Tang W, Sesh T, et al. Crowdsourcing to promote HIV testing among msm in China: a pragmatic stepped wedge randomized controlled trial of a crowdsourced intervention. Ann Glob Health. 2017;83:6.

34. Cao B, Saha PT, Leuba SI, et al. Recalling, sharing and participating in a social media intervention promoting HIV testing: a longitudinal analysis of HIV testing among MSM in China. AIDS Behav. 2019;23:1240-1249. 